\title{
NOAA/NGDC candidate models for the 11th generation International Geomagnetic Reference Field and the concurrent release of the 6th generation Pomme magnetic model
}

\author{
S. Maus ${ }^{1}$, C. Manoj ${ }^{1}$, J. Rauberg$^{2}$, I. Michaelis ${ }^{2}$, and H. Lühr ${ }^{2}$ \\ ${ }^{1}$ NOAA/NGDC and CIRES, University of Colorado \\ ${ }^{2}$ Deutsches GeoForschungsZentrum, GFZ
}

(Received December 22, 2009; Revised June 18, 2010; Accepted July 2, 2010; Online published December 31, 2010)

\begin{abstract}
The International Geomagnetic Reference Field (IGRF) is updated every five years based on candidate model submissions by research institutions worldwide. In the call for the 11 th generation of IGRF, candidates were requested for the definitive main field in 2005, the predicted main field in 2010, and the predicted secular variation from 2010 to 2015. The NOAA/NGDC candidate models for IGRF-11 were produced from parent models parameterized in the same way as the 6th generation of our Pomme magnetic model. All models were based on CHAMP satellite measurements, while Ørsted satellite measurements were used for model validation. The internal field in Pomme-6 is described by a 2nd degree Taylor time series of spherical harmonic expansion coefficients of a scalar magnetic potential. Magnetic fields of ionospheric origin are avoided by careful data selection. Instead of co-estimating magnetospheric fields, we subtract a magnetospheric field model estimated previously from a more extensive data set covering all local times. From comparison with Ørsted measurements and general considerations of magnetic field predictability, we attribute a root mean square (RMS) uncertainty of $1.3 \mathrm{nT}$ to our candidate model for the main field in 2005, $2.5 \mathrm{nT}$ to the predicted main field in 2010 and $26 \mathrm{nT} / \mathrm{a}$ to the predicted secular variation from 2010 to 2015 .
\end{abstract}

Key words: Magnetic field, magnetic satellite, magnetic reference field, main field, magnetic field modeling.

\section{Introduction}

Models of the geomagnetic main field enjoy widespread use in navigation and pointing devices. They also provide essential information on the geometry and strength of the geomagnetic field for models of the ionosphere and magnetosphere, used for example in space weather forecasting. The preferred model for engineering applications is the World Magnetic Model, WMM (Maus et al., 2010), a degree and order 12 model with fixed format, guaranteed support and release date. The preferred model of the scientific community is the International Geomagnetic Reference Field, IGRF (e.g. Maus et al., 2005), produced in an international collaborative effort under the leadership of the International Association for Geomagnetism and Aeronomy. The degree and precision of the IGRF has changed over time reflecting the increased accuracy of the underlying observing systems. While the WMM is a purely predictive model, the IGRF is adjusted retrospectively to provide more accurate "definitive" models for past epochs. In the year preceding the new 5-year epoch, a call is issued for candidates for the definitive main field at the end of the previous epoch (in this case 2005.0), a predictive main field model for the upcoming start of the new epoch (2010.0), and the predicted temporal change (secular variation) over

Copyright (c) The Society of Geomagnetism and Earth, Planetary and Space Sciences (SGEPSS); The Seismological Society of Japan; The Volcanological Society of Japan; The Geodetic Society of Japan; The Japanese Society for Planetary Sciences; TERRAPUB.

doi:10.5047/eps.2010.07.006 the upcoming epoch (2010.0-2015.0). Here we describe our respective candidates and the parent models from which these were derived.

Since the IGRF only describes the long-wavelength internal part of the geomagnetic field, candidate models are usually extracted from more extensive models which include higher-degree contributions of the internal field, seconddegree temporal derivatives (secular acceleration) and simple parameterizations of external fields of magnetospheric origins. Recent examples include the Pomme magnetic model (Maus et al., 2006), the CHAMP, Ørsted and SAC-C based CHAOS model (Olsen et al., 2006) and the GFZ Reference Internal Magnetic Model, GRIMM (Lesur et al., 2008). Such more sophisticated main field models are widely used in geomagnetism for studies of the dynamics of the earth's core (e.g. Hulot et al., 2002). Particularly accurate main field models are also required in the calibration of space-borne magnetometers, such as on the Communication and Navigation Outage Forecast Satellite C/NOFS (De La Beaujardiere, 2004).

Researchers deriving models of the main geomagnetic field can use a wide range of magnetic measurements including the network of magnetic observatories and the three satellite magnetic missions Ørsted (1999, ongoing), CHAMP (2000, ongoing) and SAC-C (2000-2004) (e.g. Lühr et al., 2009). Of the three satellites, CHAMP (Reigber et al., 2002) arguably provides the optimal data basis, with close to $100 \%$ data retrieval rate, small polar gap and dualhead star camera for low-noise attitude determination. We 
therefore base our main field models presented here entirely on CHAMP measurements and using Ørsted vector and scalar measurements for independent validation.

\section{Data Selection, Processing and Model Estima- tion}

\subsection{Data selection}

Due to the well-known spatial aliasing and crustal bias problems with ground-based observatory data, only satellite data were used in the actual model estimation. However, ground-based observatory data played an important role in data selection by providing the source of the $D_{\text {st }}$ and $a_{\mathrm{m}}$ indices.

The candidate models were entirely based on data from the CHAMP satellite. Ørsted data were processed simultaneously and used for validation of the processing steps and parent models. Ørsted data were also used in estimating the uncertainties of the Gauss coefficients.

All satellite data were sub-sampled to 20 seconds, corresponding to about $150 \mathrm{~km}$ along-track spacing. Separate data sets were compiled for mid latitudes $\left(-60^{\circ}\right.$ to $60^{\circ}$ geomagnetic latitude) and high latitudes $\left(>50^{\circ}\right.$ and $<-50^{\circ}$ geomagnetic latitude). Vector data were only used at mid latitudes. Scalar and vector data at mid latitudes were selected from the 22:00-5:00 local time sector for CHAMP and 20:00-5:00 LT for Ørsted.

CHAMP data prior to 22:00 were excluded due to the disturbing diamagnetic effect of the ambient plasma. This effect is insignificant at Ørsted altitude. Data at high latitudes were used from all local times. No exclusion for certain solar zenith angle ranges was made, since this would lead to decimated track segments. Such shortened track segments would make it difficult to compare the root mean square (RMS) residuals of neighboring tracks (see below). Specifically for CHAMP data we demanded that attitude data from dual-head star camera mode and electron density and temperature measurements were available.

In a final data selection step, the RMS values of along-track residuals against Pomme-5 (http://geomag.org/models/Pomme5.html) were sorted by longitude of the equator crossing and by date, separately for mid latitudes and for northern and southern high latitude track segments. Then the following test was carried out for each track: Its RMS value was compared with the mean RMS value of all neighboring tracks within an $8^{\circ}$ longitude range and within a one year time bin. If it was found that this track had one neighbor to the east and another neighbor to the west with an RMS at least $3 \mathrm{nT}$ smaller, then this track was declared as "noisy" and was eliminated from the data set. The threshold of $3 \mathrm{nT}$ was found by trial and error to perform well at excluding noisy data while retaining good spatial data coverage. Thus, the absolute residuals against Pomme-5 were not a selection criterion. Instead, the relative agreement with Pomme-5, as compared with neighboring tracks, was used.

\subsection{Data corrections and weighting}

The following corrections were applied to the data:

1. Estimated angular corrections for the misalignment between the magnetometer reference system and the
Table 1. Summary of data selection criteria. Here, $a_{\mathrm{m}}$ and $D_{\mathrm{st}}$ are magnetic indices, IMF- $B_{y}$ and IMF- $B_{z}$ are the vector components of the interplanetary magnetic field in Geocentric Solar Magnetospheric (GSM) coordinates at the bow shock (http://omniweb.gsfc.nasa.gov/hw.html) and $E_{\mathrm{m}}$ is the merging electric field (Kan and Lee, 1979). By mid latitudes we mean track segments covering $-60^{\circ}$ to $60^{\circ}$ geomagnetic latitude and high latitudes refers to overlapping tracks at $<-50^{\circ}$ and $>50^{\circ}$ geomagnetic latitude.

\begin{tabular}{|l|c|c|}
\hline & Mid latitudes & High latitudes \\
\hline Max $D_{\text {st }}$ & $\pm 30 \mathrm{nT}$ & $\pm 30 \mathrm{nT}$ \\
\hline Max $D_{\text {st }}$ derivative & $\pm 2 \mathrm{nT} / \mathrm{h}$ & $\pm 5 \mathrm{nT} / \mathrm{h}$ \\
\hline Max $a_{\mathrm{m}}$ & 12 & 27 \\
\hline Max $a_{\mathrm{m}} 3$ hours before & 15 & 27 \\
\hline Max IMF- $B_{y}$ & $\pm 8 \mathrm{nT}$ \\
\hline Min IMF- $B_{z}$ & \multicolumn{2}{|l}{} \\
\hline Max IMF- $B_{z}$ & $6 \mathrm{nT}$ \\
\hline Max $E_{\mathrm{m}}$ & & $0.8 \mathrm{mV} / \mathrm{m}$ \\
\hline CHAMP only: & $5 \mathrm{nT}$ & \\
\hline Max diamagnetic effect & $2 \mathrm{nT}$ & \\
\hline Max jump in diam. eff. & & \\
\hline
\end{tabular}

star tracker reference system on the CHAMP satellite are used to correct the vector data accordingly.

2. The magnetic signals of motional induction in the oceans due to the 8 major tidal constituents up to spherical harmonic degree 45 are subtracted, as predicted by Kuvshinov and Olsen (2004).

3. The signature of plasma pressure gradient currents is subtracted using the correction for the "diamagnetic effect", as proposed by Lühr et al. (2003), making use of actual electron density and temperature measurements by CHAMP.

4. A magnetospheric field model was subtracted from the data. Due to the local-time asymmetry of magnetospheric fields, day-side data have to be included in their modeling. Since day-side data are too noisy for being included in the modeling of the main field, the magnetospheric fields are best estimated in a separate precessing step. We used a revised version of the model described in Maus and Lühr (2005) which now includes the solar cycle dependence of the magnetospheric ring current. This 18-parameter model quantifies the quiet-time magnetospheric fields, modulated by the Interplanetary Magnetic Field and solar activity. Details of the model are described in Lühr and Maus (2010, this issue).

The local data density per unit area was determined, and data were then weighted accordingly in order to achieve approximate uniform spatial representation in the inversion.

\subsection{Model descriptions}

Three models were produced: The Pomme- 6 model, the parent model for our candidate for the main field in 2005 and the parent model for the main field in 2010.0 including SV 2010-2015. These three models were parameterized in a similar way, comprising:

1. The static part of the internal field to degree and order 40 for the two parent models and to degree and order 60 for Pomme-6

2. The secular variation (SV) to degree and order 16 
Table 2. Residuals of satellite observations against Pomme-6 and the two CHAMP-only parent models from which our candidates for IGRF-11 were derived.

\begin{tabular}{|c|c|c|c|c|c|c|c|c|}
\hline Data type & $X_{\mathrm{m}}(\mathrm{nT})$ & $X_{\mathrm{RMS}}(\mathrm{nT})$ & $Y_{\mathrm{m}}(\mathrm{nT})$ & $Y_{\mathrm{RMS}}(\mathrm{nT})$ & $Z_{\mathrm{m}}(\mathrm{nT})$ & $Z_{\mathrm{RMS}}(\mathrm{nT})$ & $F_{\mathrm{m}}(\mathrm{nT})$ & $F_{\mathrm{RMS}}(\mathrm{nT})$ \\
\hline \multicolumn{9}{|c|}{ Pomme-6, data range 2000.6 to 2009.67} \\
\hline CHAMP vector (mid-lat.) & 0.3 & 3.4 & 0.2 & 3.6 & -0.2 & 3.0 & 0.1 & 2.8 \\
\hline CHAMP scalar (global) & & & & & & & -0.2 & 3.1 \\
\hline Ørsted vector (mid-lat.) & -0.6 & 5.9 & -0.7 & 5.8 & 0.3 & 4.7 & 0.0 & 3.0 \\
\hline Ørsted scalar (global) & & & & & & & 0.9 & 2.7 \\
\hline \multicolumn{9}{|c|}{ Parent model 2005, data range 2003.5 to 2006.5} \\
\hline CHAMP vector (mid-lat.) & 0.2 & 3.3 & 0.2 & 3.8 & -0.1 & 2.7 & 0.1 & 2.5 \\
\hline CHAMP scalar (global) & & & & & & & -0.2 & 3.2 \\
\hline Ørsted scalar (global) & & & & & & & 1.1 & 2.6 \\
\hline \multicolumn{9}{|c|}{ Parent model 2010, data range 2006.5 to 2009.67} \\
\hline CHAMP vector (mid-lat.) & 0.3 & 3.1 & 0.1 & 3.0 & -0.4 & 2.2 & 0.1 & 2.4 \\
\hline CHAMP scalar (global) & & & & & & & -0.1 & 3.1 \\
\hline Ørsted scalar (global) & & & & & & & 1.4 & 2.4 \\
\hline
\end{tabular}

3. The secular acceleration (SA) to degree and order 16. For Pomme- 6 separate coefficients of the SA are provided for the periods before and after the mid point of the data interval in 2005.0. This temporal representation with two sets of SA coefficients and a single set of SV coefficients was first introduced in Pomme-4 (http://geomag.org/models/pomme4.html).

4. A daily varying degree-1 external field, as proposed by Olsen et al. (2006), represented by a single value of the strength of the axial dipole in Solar-Magnetic, SM, frame for every $24 \mathrm{~h}$ interval.

The model coefficients were estimated in a non-iterative least-squares approach, where the information from the scalar data was linearized using Pomme-5. The SV was regularized starting at degree and order 14 , while the SA coefficients were damped for degrees 9 and higher, as described in Maus et al. (2006).

The data intervals for Pomme-6 and the IGRF parent models were chosen as follows:

1. For Pomme-6 the entire available CHAMP data were used from 2000.6 to 2009.7 .

2. To estimate the main field in 2005.0, we chose satellite data from 2003.5 to 2006.5 . The primary parent model was produced from CHAMP data only. A second parent model was produced by including Ørsted scalar data. This model was only used for validation and to estimate the uncertainty of the coefficients of the first model. For this purpose it would have been better to have a completely independent model of pure Ørsted data. However, since Ørsted stopped supplying vector measurements after 2003, it was not possible to produce an accurate model from Ørsted-only data.

3. To predict the main field in 2010.0 and the secular variation 2010-2015, we used the last three years of available data, spanning 2006.5-2009.7. Again, the primary parent model was based only on CHAMP data, while a second model including Ørsted data was used for estimating Gauss coefficient uncertainties.

\subsection{Derivation of candidate model coefficients and un- certainties}

The following procedures were used to derive the three candidate products and the uncertainties of the coefficients:

1. The main field in 2005.0 was simply taken as the static coefficients at the center of the CHAMP-only model. Their uncertainty was estimated by taking the difference to the corresponding coefficients of the model including Ørsted data, and multiplying the difference by three. The ad-hoc rationale for the factor three is that the Ørsted data had 1/3 weight in the combined model, so a "pure" Ørsted model would presumably exhibit three times the observed deviation to the CHAMP-only model. A minimum uncertainty of $0.01 \mathrm{nT}$ was imposed in order to reflect the added uncertainty due to rounding errors. The total RMS uncertainty in the vector field averaged over the Earth surface can be inferred from the square root of the total Lowes/Mauersberger power. It amounts to $1.3 \mathrm{nT}$.

2. The main field in 2010.0 was predicted by evaluating the Taylor time series using secular variation and secular acceleration coefficients for the date 2010.0. The uncertainties were evaluated in the same way as for the 2005 model. The total RMS uncertainty is estimated as $2.5 \mathrm{nT}$

3. Studies of fore- and hind-cast of the secular variation of the geomagnetic field (e.g. Maus et al., 2008) suggest that the predictive quality of the secular acceleration seems to be quite limited. Taking a pessimistic view, we therefore provide the SV of the parent model at the end of the data interval (2009.67) as our best estimate of the SV 2010-2015. The primary uncertainty in SV forecast lies in the unpredictable behavior of the SA. As an estimate of uncertainty combining measurement and prediction uncertainties, we therefore take the difference between the SV of the CHAMP model in 2009.67 and the forward-extrapolated SV (using the SA coefficients) of the combined model to 2012.5. The latter accounts for unpredictable changes in the true $\mathrm{SV}$, in addition to the uncertainty in estimating the present SV. This approach leads to a large (but real- 

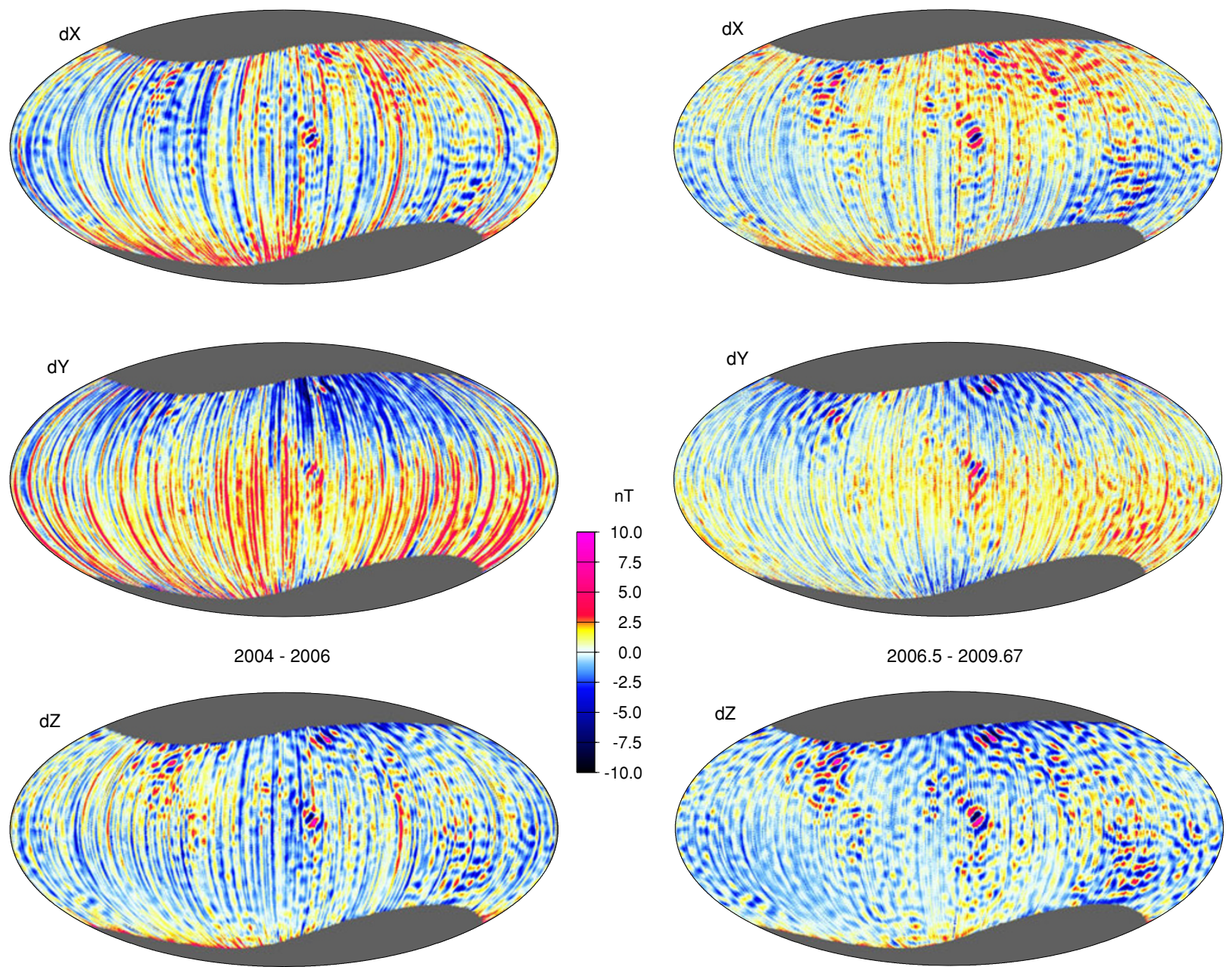

Fig. 1. Vector component residuals of the two CHAMP-only parent models against the data that were used to estimate the model coefficients. Longitude range is $-180^{\circ}$ to $+180^{\circ}$. The small-scale "bubbly" features are due to unmodeled crustal field beyond the model cut-off at degree 40 . These features are stronger in the column on the right side due to the lower altitude of the CHAMP satellite. Furthermore, one can see that the residuals are significantly more noisy (striped patterns) in the earlier years, represented in the left column. This solar activity dependent difference is clearly visible despite the rigorous data selection criteria employed.

istic) uncertainty estimate of $26 \mathrm{nT} / \mathrm{a}$. This means that we expect the cumulative RMS error of the IGRF to reach $130 \mathrm{nT}$ by the end of the model epoch in 2015 .

\subsection{Test models with extended-degree secular varia- tion}

Presently, the predictive secular variation of the IGRF only extends to degree and order 8 , a relict of the presatellite era in which the high-degree SV was impossible to observe. With the present satellite coverage, it is possible to obtain an un-damped estimate of the SV to degree 13 from only 3 years of data. It therefore would have seemed obvious to extend the predictive SV from degree 8 to degree 13 , already for the epoch 2005-2010. However, this suggestion was dismissed in 2004 and again in 2009, even though a study by Silva et al. (2010, this issue), presented at IAGA2009 in Sopron, demonstrated that the SV coefficients at degrees 9-12 of the parent models for IGRF-2005 provided a significantly better estimate of the SV in the 2005-2010 epoch than the current practice of setting these SV coefficients to zero. In order to further strengthen the case for extending the predictive SV to degree 13 in 2014 for IGRF-12, all groups were asked to provide extended-degree test ver- sions of their SV candidate models. These extended-degree test models can then be compared with the true SV in subsequent years in order to assess the reliability of high-degree SV predictions.

Our two test-candidates with extended-degree SV coefficients are derived from the second parent model at the end of the data interval (2009.67):

1. The extended-degree model corresponding to our candidate model submission for SV-2010-2015 based only on CHAMP data

2. The extended-degree model estimated from a combination of CHAMP and Ørsted data, which was used for the uncertainty estimation of IGRF-2010 and SV2010-2015.

The uncertainty of the two extended models is estimated in the same way as for the SV to degree 8 . The higher degrees contribute very little to the overall uncertainty, which has the same RMS of $26 \mathrm{nT}$ as the truncated model. 

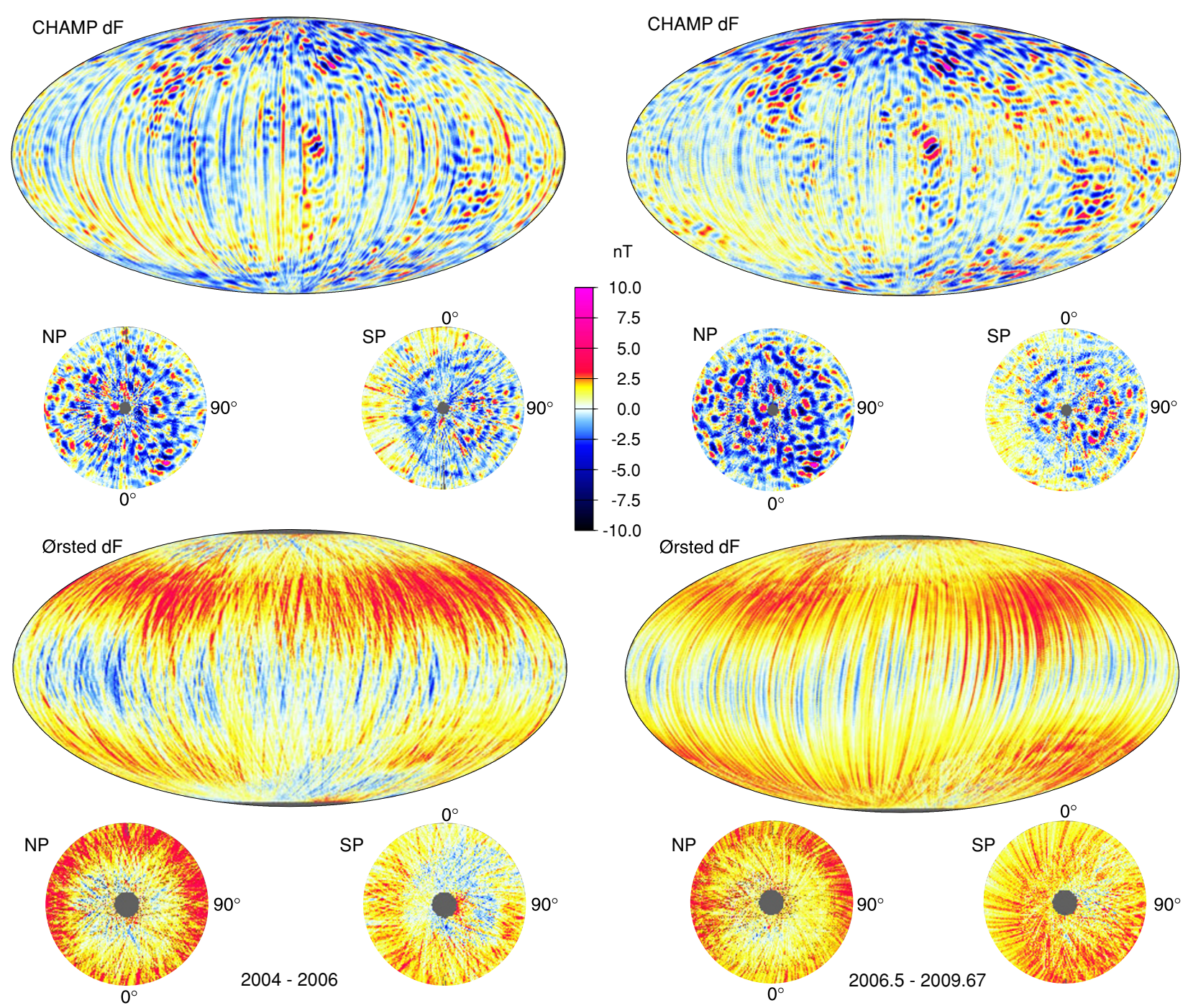

Fig. 2. Scalar residuals of the two CHAMP-only parent models against CHAMP scalar data (top row) and independent Ørsted scalar data (bottom row).

\section{Results}

Pomme-6 and the IGRF parent models were first validated against the CHAMP data from which they were produced. Subsequently, they were validated against Ørsted scalar data. The latter constitutes a truly independent validation since Ørsted data was neither used in the production of these models, nor in the production of Pomme-5 which played a role as a reference model in the data selection and in the linearization of the inverse problem. Table 1 displays the residuals of satellite data against Pomme- 6 and the two CHAMP-only parent models from which our candidates for IGRF-11 were derived. A slight reduction in RMS in all vector components is observable from the earlier to the later period due to the declining solar cycle. The Ørsted scalar data indicate a systematic bias of $1.4 \mathrm{nT}$ against the later parent model, and a slightly lower average bias of $0.9 \mathrm{nT}$ against Pomme-6, where the entire period since 2000 is taken into account. The zero scalar residual of the Ørsted vector data for the earlier period indicates that the discrepancy only emerges after the failure of the Ørsted vector magnetometer. Note that the residuals are not weighted for spatial coverage. Thus, the contribution from high-latitude measurements is disproportionately large. All residuals are calculated as measurement minus model value.

The global distributions of CHAMP vector component residuals against the two CHAMP-only parent models are displayed in Fig. 1. The scalar residuals for CHAMP and independent Ørsted data are shown in Fig. 2. The Ørsted residuals show a consistent positive offset of about $1 \mathrm{nT}$, meaning that Ørsted measures a stronger field than the models predict for that altitude. This is consistent with the derived mean values in Table 2 .

To investigate whether the difference between CHAMP and Ørsted residuals is due to a genuine difference in field strength, the mean residual against Pomme- 6 is plotted in Fig. 3 as a time series. A genuine effect should be persistent and could be solar cycle dependent. Instead, the CHAMP and Ørsted residuals are in good agreement until 2003, and subsequently they deviate by about $1.5 \mathrm{nT}$, indicating a possible baseline shift of one of the scalar measurements. While the Ørsted residuals show a prominent 800-day periodicity coupled to its local time variation, a corresponding 130-day local time cycle is not visible in the CHAMP data.

Plotting the mean residual from the time after 2005.0 against latitude, as shown in Fig. 4, reveals that the dif- 


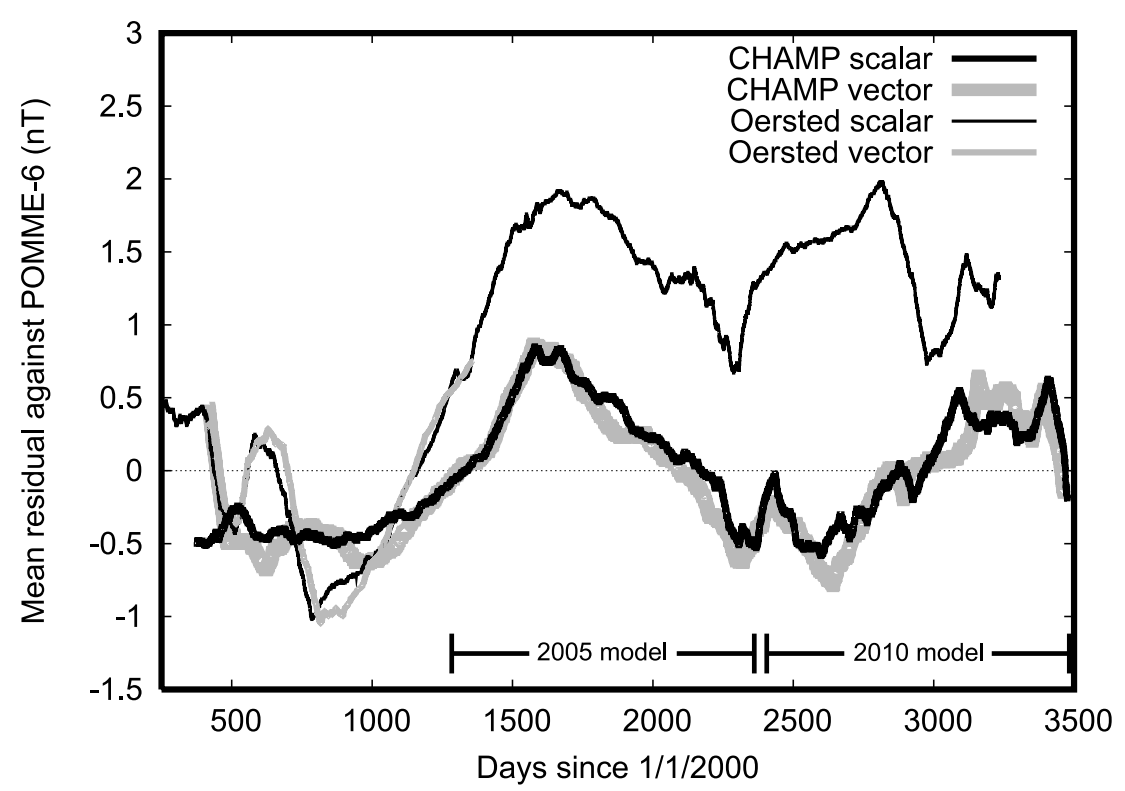

Fig. 3. Evolution of the mean residual against Pomme-6 over time. After 2004.0 (day 1460) we find a significant offset between the residuals of the two satellites. In addition, the Ørsted residuals indicate a possible 800-day local time variation.

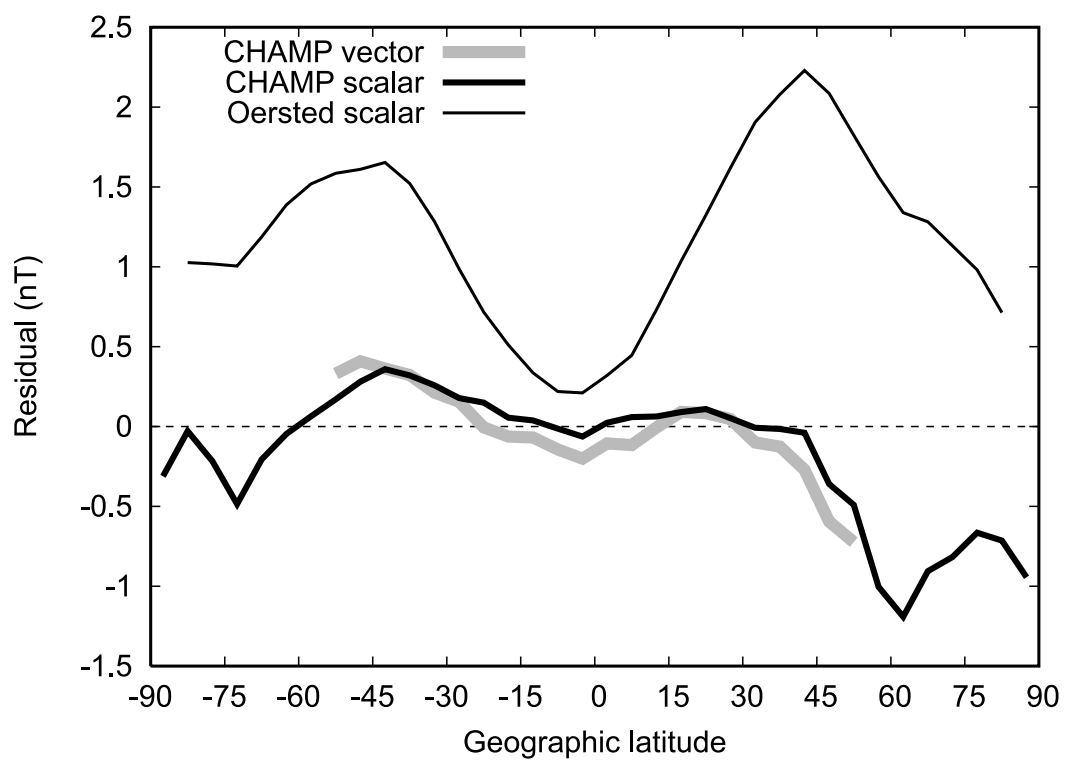

Fig. 4. Mean residuals against Pomme-6 versus geographic latitude, averaged over all longitudes in the time interval 2005.0 to 2009.67 . There is only a marginal difference between the curves plotted versus geographic or geomagnetic latitude.

ference between Ørsted and CHAMP is almost negligible at the equator, and peaks at about $40^{\circ}$ latitude. However, whether the shift is due to Ørsted or CHAMP instrumentation is difficult to deduce from the data available.

\section{Summary and Conclusions}

Pomme-6 and three candidates for IGRF-11 have been derived from CHAMP satellite data and validated with Ørsted satellite data. All models and the software are available at our web site http://geomag.org/models/pomme6.html.

Judging from the small residuals of the data against the models, an overall accuracy of the order of $1 \mathrm{nT}$ per component (at least at mid latitudes) has likely been achieved by our main field candidate for 2005 . For the prediction of the main field at epoch 2010 somewhat larger and for the SV 2010-2015 much larger inaccuracies must be assumed, due to inherent problems with forecasting the future evolution of the geomagnetic field. An important question is whether the presently observed secular acceleration can be used to extrapolate the SV to the center of the upcoming epoch. Our analysis of past field behavior indicates that this is highly speculative. We have therefore taken the conservative approach of using the modeled SV at the end of the data period as an estimate of the SV for the upcoming epoch.

As an interesting secondary result, we find a systematic difference between the field strengths predicted by the CHAMP model and the Ørsted measurements at a different altitude. The Ørsted residuals appear to exhibit an additional 800 day local time periodicity. These scalar residuals 
show a systematic latitude variation. Peak values occur just at latitudes $\left(\sim 40^{\circ}\right)$ where the magnetic field of the ring current does not contribute to the field magnitude. However, the discrepancy does not exhibit a behavior over time that supports the existence of a genuine difference in the ambient field strength. We are therefore not sure whether the differences are due to a deficit in external field characterization or a deviation between the scalar magnetic field readings of the two spacecraft.

Acknowledgments. We thank Brian Hamilton and Angelo DeSantis for their careful reviews and helpful suggestions. The CHAMP mission is supported by the German Aerospace Center (DLR) and the Federal Ministry of Education and Research. The Ørsted Project was made possible by extensive support from the Danish Government, NASA, ESA, CNES and DARA.

\section{References}

De La Beaujardiere, O., C/NOFS: a mission to forecast scintillations, $J$. Atmos. Sol.-Terr. Phys., 66, 1573-1591, 2004.

Hulot, G., C. Eymin, B. Langlais, M. Mandea, and N. Olsen, Small-scale structure of the geodynamo inferred from Ørsted and Magsat satellite data, Nature, 416, 620-623, 2002.

Kan, J. R. and L. C. Lee, Energy coupling function and solar wind magnetosphere dynamo, Geophys. Res. Lett., 6, 577-580, 1979.

Kuvshinov, A. and N. Olsen, 3-D modelling of the magnetic fields due to ocean tidal flow, in Earth Observation with CHAMP, Results from Three Years in Orbit, edited by C. Reigber, H Lühr, P. Schwintzer, and J. Wickert, 359-365, Springer, Berlin, 2004.

Lesur, V., I. Wardinski, M. Rother, and M. Mandea, GRIMM: the GFZ Reference Internal Magnetic Model based on vector satellite and observatory data, Geophys. J. Int., 173, 382-394, doi:10.1111/j.1365246X.2008.03724.x, 2008.

Lühr, H. and S. Maus, Solar cycle dependence of quiet-time magnetospheric currents and a model of their near-Earth magnetic fields, Earth Planets Space, 62, this issue, 843-848, 2010.

Lühr, H., M. Rother, S. Maus, W. Mai, and D. Cooke, The diamagnetic effect of the equatorial Appleton anomaly: Its characteristics and impact on geomagnetic field modeling, Geophys. Res. Lett., 30, 1906, doi:10.1029/2003GL017407, 2003.

Lühr, H., M. Korte, and M. Mandea, The recent geomagnetic field and its variations, in Geomagnetic Field Variations, edited by K.-H. Glassmeier, H. Soffel, and J. Negendank, 25-63, Springer-Verlag, BerlinHeidelberg, 2009.

Maus, S. and H. Lühr, Signature of the quiet-time magnetospheric magnetic field and its electromagnetic induction in the rotating Earth, Geophys. J. Int., 162, 755-763, doi:10.1111/j.1365-246X.2005.02691.x, 2005.

Maus, S., S. Macmillan, T. Chernova, S. Choi, D. Dater, V. Golovkov, V. Lesur, F. Lowes, H. Lühr, W. Mai, S. McLean, N. Olsen, M. Rother, T. Sabaka, A. Thomson, and T. Zvereva, The 10th-Generation International Geomagnetic Reference Field, Geophys. J. Int., 161, 561-565, doi:10.1111/j.1365-246X.2005.02641.x, 2005.

Maus, S., M. Rother, C. Stolle, W. Mai, S. Choi, H. Lühr, D. Cooke, and C. Roth, Third generation of the Potsdam Magnetic Model of the Earth (Pomme), Geochem. Geophys. Geosyst., 7, Q07008, doi:10.1029/2006GC001269, 2006.

Maus, S., L. Silva, and G. Hulot, Can core-surface flow models be used to improve the forecast of the Earth's main magnetic field?, J. Geophys. Res., 113, B08102, doi:10.1029/2007JB005199, 2008.

Maus, S., S. Macmillan, S. McLean, B. Hamilton, A. Thomson, M. Nair, and C. Rollins, The US/UK World Magnetic Model for 2010-2015, NOAA Technical Report NESDIS/NGDC, 2010.

Olsen, N., H. Lühr, T. J. Sabaka, M. Mandea, M. Rother, L. TøffnerClausen, and S. Choi, CHAOS-A Model of Earth's Magnetic Field derived from CHAMP, Ørsted, and SAC-C magnetic satellite data, Geophys. J. Int., 166, 67-75, doi:10.1111/j.1365-246X.2006.02959.x, 2006. Reigber, C., H. Lühr, and P. Schwintzer, CHAMP mission status, Adv. Space Res., 30, 129-134, 2002.

Silva, L., S. Maus, G. Hulot, and E. Thébault, On the possibility of extending the IGRF predictive secular variation model to a higher SH degree, Earth Planets Space, 62, this issue, 815-820, 2010.

S. Maus (e-mail: Stefan.Maus@noaa.gov), C. Manoj, J. Rauberg, I. Michaelis, and H. Lühr 